PREPARED FOR SUbMISSION TO JINST

\title{
Design of a Nondestructive Two-in-One Instrument for Measuring the Polarization and Energy Spectrum at an X-ray FEL Facility
}

\author{
Qingmin Zhang, ${ }^{a}$ Bangjie Deng, ${ }^{a}{ }^{1}$ Yuanmiaoliang Chen, ${ }^{a}$ Bochao Liu, ${ }^{a}$ Shaofei Chen, ${ }^{a}$ Jinquan Fan, ${ }^{a}$ \\ Lie Feng, ${ }^{b}$ Haixiao Deng, ${ }^{b, 2}$ Bo Liu, ${ }^{b}$ Dong Wang ${ }^{b}$ \\ ${ }^{a}$ Xi'an Jiaotong University, Xi'an, 710049, China \\ ${ }^{b}$ Shanghai Institute of Applied Physics, Chinese Academy of Sciences, Shanghai, 201800, China \\ E-mail: dengbangjie@foxmail.com, denghaixiao@sinap.ac.cn
}

\begin{abstract}
Aвstract: The free electron laser (FEL), as a next-generation light source, is an attractive tool in scientific frontier research because of its advantages of full coherence, ultra-short pulse duration, and controllable polarization. Owing to the demand of real-time bunch diagnosis during FEL experiments, precise nondestructive measurements of the polarization and X-ray energy spectrum using one instrument are preferred. In this paper, such an instrument based on the electron time-of-flight technique is proposed. By considering the complexity and nonlinearity, a numerical model in the framework of Geant 4 has been developed for optimization. Taking the Shanghai Soft X-ray FEL user facility as an example, its measurement performances' dependence on the critical parameters was studied systematically, and, finally, an optimal design was obtained, achieving resolutions of $0.5 \%$ for the polarization degree and $0.3 \mathrm{eV}$ for the $\mathrm{X}$-ray energy spectrum.
\end{abstract}

KeYwords: X-ray FEL, e-TOF, Polarization, Energy spectrum

ArXIV EPrint: 1702.06652

${ }^{1}$ Corresponding author.

${ }^{2}$ Corresponding author. 


\section{Contents}

1 Introduction $\quad 1$

2 Method description $\quad 2$

2.1 Polarization measurement 2

$2.2 E_{p h}$ spectrum measurement 3

3 Overall design $\quad 3$

4 Numerical modeling $\quad 5$

4.1 Model description $\quad 6$

$\begin{array}{lll}4.2 & \text { Simulation } & 7\end{array}$

5 Dependence analysis $\quad 8$

$\begin{array}{llr}5.1 & \text { Polarization measurement } & 8\end{array}$

$\begin{array}{lll}5.2 & E_{p h} \text { spectrum measurement } & 11\end{array}$

6 Discussion and conclusion $\quad 13$

\section{Introduction}

Recently, owing to their irreplaceable advantages of high brightness[1], fully transverse coherence[2, 3], ultrashort pulse duration [4] and well-defined polarization [5-7], several soft and hard X-ray free electron laser (FEL) user facilities, as the next-generation light source, have been designed, under construction, or in operation; these include LCLS (SLAC, USA) [8], SACLA (SPRING8, Japan)[9], the European XFEL (Hamburg, Germany)[10], FERMI (Trieste, Italy)[11], and SXFEL (Shanghai, China)[12]. Currently, SXFEL with a designed wavelength of $8.8 \mathrm{~nm}$ is under commissioning as an FEL test facility. Furthermore, a project upgrading SXFEL to a user facility has been launched, in which the electron beam's energy will be boosted from 0.84 to $1.6 \mathrm{GeV}$, to cover the water window or even the magnetic window[13]. According to the baseline design of the SXFEL user facility, each X-ray pulse contains more than $10^{12}$ photons with $\sim 100 \mathrm{fs}$ pulse duration and controllable polarization[12].

It is well known that high-resolution measurement of the polarization properties and X-ray energy spectrum are strongly demanded in soft X-ray magnetic circular dichroism spectroscopy. Meanwhile, the SXFEL user facility requires noninvasive measurements of polarization and $E_{p h}$ with $0.5 \%$ and $0.5 \mathrm{eV}$ precision, respectively. However, conventional optical methods, in which transmission polarizers or multilayer reflectors are used[14], are no more suitable owing to soft X-ray's strong absorption in the interaction materials. At FERMI, with an $\mathrm{X}$-ray energy range from 22.9 to $47.6 \mathrm{eV}$, FEL polarization was measured by using three methods: EUV light fluorescence, VUV optics, and photoelectron angular distributions [15]. The electron time-of-flight (e-TOF) technique is utilized in the third method, so it is routinely called "e-TOF based." It is a noninvasive method because only a small fraction of photons interact with the rarefied gas in the e-TOF instrument and the others go through without any change. However, high resolution can be achieved because of the beam's high intensity. In contrast, the other two methods have more serious impact on the photon beam owing to their transmission 
optics and phase retarders[15]. Besides, the e-TOF-based instruments have been successfully applied in a Polarization monitor at an X-ray FEL[6, 7]. It is worth mentioning that e-TOF-based X-ray spectroscopy has been applied widely for many years and that its time resolution has been improved from picoseconds[16-18] to femtoseconds[19-21], which has paved the road to precisely measure the photon energy spectrum for ultra-short $\mathrm{X}$-ray FEL pulses.

Accordingly, the e-TOF-based method can be utilized to simultaneously measure the polarization and energy spectrum of a soft X-ray FEL. In this paper, by considering the complexity and nonlinearity, a numerical model in the framework of Geant4[22] is established for simulating such a two-in-one instrument, which is based on the e-TOF technique for simultaneously measuring the polarization and energy spectrum. With the SXFEL user facility taken as an example, its systematic design is devised and its optimization is performed. Finally, our study shows that a resolution of $0.5 \%$ in polarization degree and $0.3 \mathrm{eV}$ in X-ray energy spectrum can be obtained with the optimal design.

\section{Method description}

For each X-ray FEL pulse, photoelectrons are produced by X-ray photon photoionizations with target atoms. By measuring their angular distribution and drift time, one can simultaneously measure the polarization properties and $E_{p h}$ spectrum of the X-ray FEL. In this section, measurement principles will be described in detail.

\subsection{Polarization measurement}

The electric vector $(\vec{E})$ of completely polarized light can be expressed in the form

$$
\begin{aligned}
\vec{E} & =\underbrace{A_{x} \cos \omega t \cdot \vec{e}_{x}+A_{y} \cdot \cos (\omega t+\Delta \varphi) \cdot \vec{e}_{y}}_{\text {linearly polarized part }} \\
& =\underbrace{\left(A_{x}+A_{y} \sin \Delta \varphi\right) \cos (\omega t) \cdot \vec{e}_{x}+A_{y} \cos \Delta \varphi \cos (\omega t) \cdot \vec{e}_{y}}_{\text {circularly polarized part }} \\
& +\underbrace{\left(-A_{y} \sin \Delta \varphi\right) \cdot\left[\cos (\omega t) \cdot \vec{e}_{x}+\sin (\omega t) \cdot \vec{e}_{y}\right]} .
\end{aligned}
$$

As shown in Eq. 2.1, completely polarized light can be decomposed into linearly polarized light $\left(E_{\text {lin }}\right)$ and circularly polarized light $\left(E_{c i r}\right)$, which means that linear polarized light and circularly polarized light can be used to describe the polarization properties of completely polarized light. The photoelectron angular distribution of linearly polarized photons is different from that of circularly polarized or nonpolarized photons; hence, the polarization properties of an X-ray FEL can be inferred by using these angular distribution.

For $s$ shells, the photoelectron angular distribution in the plane perpendicular to photon momentum direction (in the detection plane) is described precisely enough by the dipole approximation[23-25]. For a completely polarized X-ray FEL, a normalized probability distribution of photoelectrons from $s$ shells in the plane perpendicular to the photon direction is $[6,15]$

$$
p(\theta)=\frac{1}{2 \pi}+P_{\text {lin }} \cdot \frac{3 \beta}{2 \pi(4+\beta)} \cos [2(\theta-\Psi)],
$$

where $P_{l i n}$ is the linear polarization degree, $\Psi$ is the polarization angle of linearly polarized photons, $\theta$ is the angle between the photoelectrons momentum and the polarization direction, and $\beta$ is the dipole parameter related to the gas target species.

However, for $p$ shells, the differential photoelectric cross section cannot be described by the electric dipole approximation, which results in complexity for data analysis. However, electrons from $p$ shells can be excluded by the longer time of flight, which is related to their lower kinetic energy. 
According to Eq. 2.2, $p(\theta)$ can be obtained by fitting an electron angular distribution with $p(\theta)=$ $A+B \cdot \cos [2(\theta-C)]$. Then, the linear (circular) polarization degree $P_{\text {lin }}\left(P_{\text {cir }}\right)$ would be

$$
\left\{\begin{array}{l}
P_{\text {lin }}=\frac{B}{A} \cdot \frac{3 \beta}{4+\beta}, \\
P_{\text {cir }}=\sqrt{1-P_{\text {lin }}^{2}}
\end{array}\right.
$$

and polarization angle would be $\Psi=C$.

\section{$2.2 \quad E_{p h}$ spectrum measurement}

Photon energy can also be derived by the time of flight, since the relation between photoelectron energy $\left(E_{e}\right)$ and photon energy $\left(E_{p h}\right)$ is

$$
E_{p h}=E_{e}+E_{b},
$$

where $E_{b}$ represents the electron's binding energy for a given shell, which varies from gas to gas[26, 27]. By measuring the electron's flight time for a given drift length $L, E_{p h}$ can be determined with

$$
E_{p h}=\frac{m_{e} L^{2}}{2 \Delta t^{2}}+E_{b}
$$

where $\Delta t$ is the photoelectron flight time and $m_{e}$ is the electron's rest mass.

As mentioned above, $E_{b}$ varies with target gases. However, because the angular distribution of Auger electrons is different from that of photoelectrons $\left(E_{k, p h}\right)$, gas selection should avoid overlap with the kinetic energy of Auger electrons $\left(E_{k, \text { Auger }}\right)$, which is

$$
E_{k, \text { Auger }}=E_{i}-E_{j}-E_{k},
$$

where $E_{i}, E_{j}$, and $E_{k}$ represent the electron binding energies for shell $i, j$ and $k$, respectively. These properties can be found in References [25] and [28].

By making use of pulse waveform analysis and earliest arrival time measurement, one can obtain the flight time spectrum for photoelectrons, from which $E_{p h}$ spectrum can be derived according to Eq. 2.6.

\section{Overall design}

According to the physical principles described above, the photoelectron angular distribution can be measured by placing detectors in the detection plane and their energy spectrum can be obtained by using the time of flight. The instrument is designed to contain three subsystems: a detector, electronics, and data analysis, as demonstrated in Fig. 1.

The detector system is composed of a differential vacuum system and a given number of identical e-TOF detectors for photoelectron generation, drifting, and detection. The gas target is located at the center of the instrument for photoelectric reaction, being uniformly surrounded by e-TOF detectors. Each e-TOF detector is an electron multiplier equipped with a collimation tube. Micro-channel plates (MCPs) are preferred as electron multipliers owing to their timing resolution of hundreds of picoseconds because of their thinner micro-channels and better response linearity because of their numerous independent micro-channels and electron dispersive arrival at the MCP in space. In addition, circular truncated cone collimation tubes were designed for better placement. Following the X-ray FEL beam's arrival, photoelectrons are generated in the central volume, drift through the collimation tubes, and are finally detected by electron detectors. Given statistical errors, the pressure in the central volume should be fairly high for generating enough photoelectrons, while its surrounding volume 


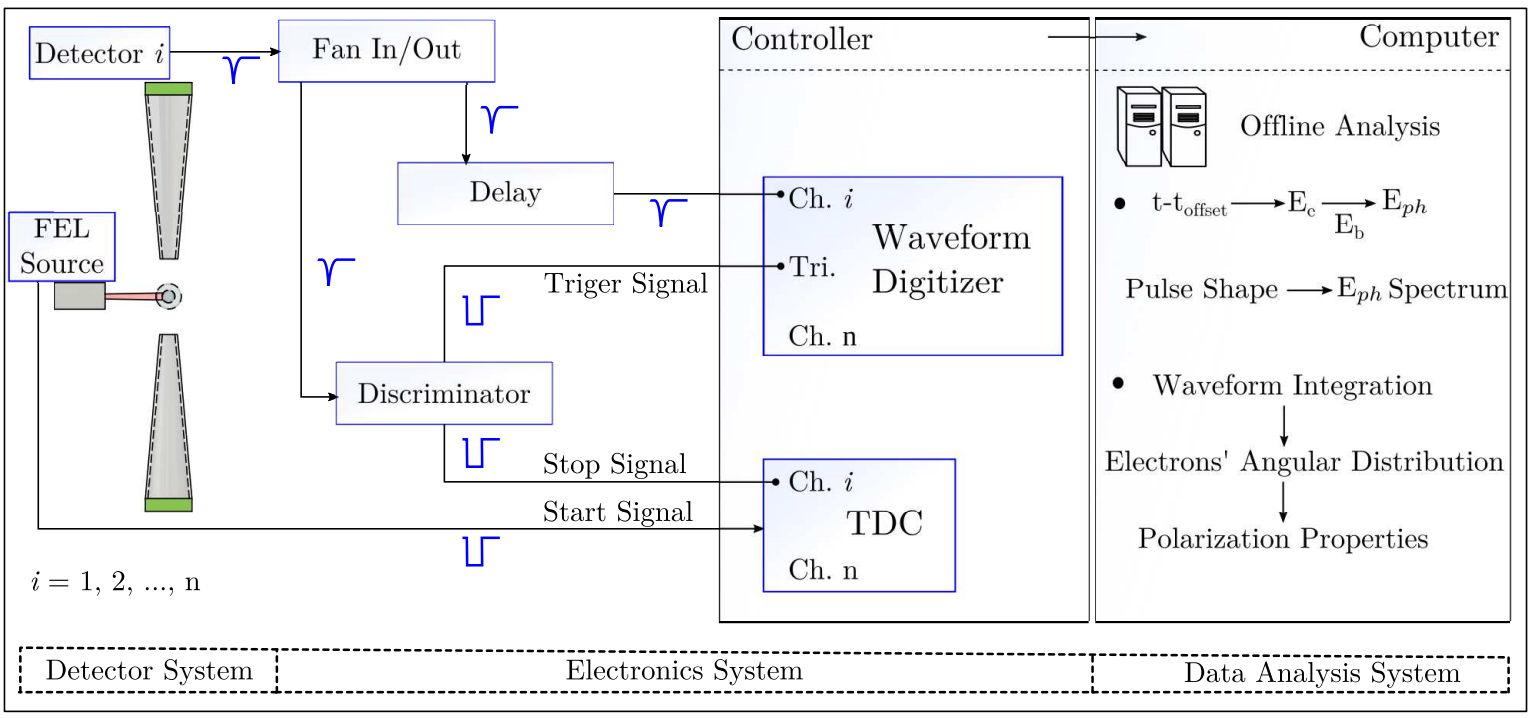

Figure 1. Block diagram of the apparatus.

should be at very low pressure to minimize electron loss and scattering during drifting and to satisfy the detectors' working pressure requirement. Accordingly, a differential vacuum system (DVS) was adopted. A small difference between the target gas and that of the drifting environment is preferable to avoid complicated DVS design.

The electronics system was designed to record the flight time and signal waveform of photoelectrons by using a time-to-digital converter (TDC) unit and a waveform digitizer for all detectors, respectively. For each $\mathrm{X}$-ray FEL pulse, the TDC is triggered by a start signal supplied by The timing system of the FEL source and stopped by a signal from a discriminator. Meanwhile, the stop signal is also transferred to the waveform digitizer for signal shape recording. A proper delay for the detection signal transferred to the waveform digitizer is introduced for synchronization in order to record the full signal shape. The signal shape can be integrated to obtain the signal charge, which is proportional to the number of photoelectrons. Then, the photoelectron angular distribution is obtained to conclude attainment of the polarization properties. By combining the signal shape and flight time, the photoelectron flight time spectrum can be determined.

The data analysis system was designed to analyze waveform time series data, from which the photoelectron flight time spectrum can be derived by using pulse shape analysis methods. Furthermore, the $E_{p h}$ spectrum can be obtained according to Eq. 2.6 after an offset time correction. It is reasonably assumed that there is no saturation effect for the detector's response owing to the moderate number of detected electrons and that the pulse shape for each electron is essentially the same. Therefore, the signal's integration charge for each channel $\left(Q_{i}, i=1,2, \ldots, 16\right)$ is proportional to the number of detected photoelectrons. Then the X-ray FEL's polarization properties $\left(P_{\text {lin }}\right.$ and $\left.P_{\text {cir }}\right)$ can be obtained by fitting $Q_{i}$ with $p(\theta)=A+B \cos 2[(\theta+C)]$ and applying Eq. 2.3 to Eq. 2.4. Based on the assumptions mentioned above, a linear model was proposed to analyze the signal waveform to obtain the flight time spectrum. The sampled signal pulse $P\left(i \Delta t_{s}\right)(i=1,2, \ldots, n)$ with sampling time interval $\Delta t_{s}$ can be described as

$$
P=\left[P_{1}, P_{2}, \ldots, P_{i}, \ldots, P_{n}-1, P_{n}\right]
$$

Because $\Delta t_{s}$ for the waveform digitizer is much larger than the realistic interval of electron arrival time, the sampled signal should be interpolated to acquire a smaller time interval $\Delta t_{i}$ for decomposition. The interpolation 
signal pulse shape $I\left(j \Delta t_{i}\right),(j=1,2, \ldots, m)$ can be obtained by interpolating the sampled signal $P\left(i \Delta t_{s}\right)$ in a small interpolation time interval $\Delta t_{i}$. Obviously, $m \geq n$ is required and $n \Delta t_{s}$ is much longer than the typical width of the whole signal. Commonly, the amplitude of a single photoelectron's charge signal $\left(S_{0, j}\right)$ with time interval $\Delta t_{i}$ in a simulation time $t=j \Delta t_{i}(j=1,2, \ldots, m)$ can be described by the log-normal distribution $f(t)$ in the form

$$
f(t)=e^{-\frac{1}{2} \cdot\left(\frac{\log (t / \tau)}{\sigma_{s}}\right)^{2}}
$$

where $\tau$ and $\sigma_{s}$ define the signal's location and scale, respectively[29]. Therefore, the signal's pulse shape ( $\left.\mathbf{S}_{\mathbf{0}}\right)$ for a single electron without delay can be described digitally as follows:

$$
\left\{\begin{array}{l}
\mathbf{S}_{\mathbf{0}}=\left[S_{0,1}, S_{0,2}, \ldots, S_{0, j}, \ldots, S_{0, m}\right]^{T} \\
S_{0, j}=f\left(j \Delta t_{i}\right) \quad(j=1,2, \ldots, m) .
\end{array}\right.
$$

Thus, the pulse shape for a single electron with a delay of $k \Delta t_{i}(k=0,1,2, \ldots, m-1)$ can be expressed digitally as

$$
\left\{\begin{array}{l}
\mathbf{S}_{\mathbf{k}}=\left[S_{k, 1}, S_{k, 2}, \ldots, S_{k, j}, \ldots, S_{k, m}\right]^{T} \\
S_{k, j}=f\left((j-k) \Delta t_{i}\right) \quad(j=1,2, \ldots, m) .
\end{array}\right.
$$

Then, signal matrix for a series of single electrons with a delay $k \Delta t$ can be written as

$$
\mathbf{S}_{m \times m}=\left[\mathbf{S}_{\mathbf{0}}, \mathbf{S}_{\mathbf{1}}, \ldots, \mathbf{S}_{\mathbf{m}-\mathbf{2}}, \mathbf{S}_{\mathbf{m}-\mathbf{1}}\right]
$$

In addition, the number of electrons for each bin can be expressed as

$$
\mathbf{C}_{m \times 1}=\left[C_{0}, C_{1}, \ldots, C_{m-2}, C_{m-1}\right]^{T}
$$

Thus,

$$
\mathbf{I}=\mathbf{S} \cdot \mathbf{C}+\mathbf{e},
$$

where $\mathbf{e}$ is the error term resulting from noise. Therefore, the number of electrons for each timing bin is obtained by solving Eq. 3.9, and, namely, the electron's flight time spectrum can be obtained. A least-squares method is chosen for optimization in solving for $\mathbf{C}$ in Eq. 3.9. The estimated number of photoelectrons, $\hat{\mathbf{C}}$, can be obtained by using various optimization algorithms with constraints $\left(C_{j} \geq 0, j=0,1, \ldots, m-1\right)$, such as the conjugate gradient method[30], the Broyde-Fletcher-Goldfarb-Shanno method[31], etc. Finally, the $E_{p h}$ spectrum can be derived by using Eq. 2.6.

\section{Numerical modeling}

Because the instrument's performance is influenced by many factors and photoelectron interactions with the target gas cannot be well described analytically, it is impossible to optimize the design analytically. Hence, a numerical simulation model will be established in the framework of Geant4, which is a Monte Carlo nuclear physics simulation software package[22]. To simulate polarized photon interactions with the target gas, the Livermore Polarized Physics Model with G4EMLOW-6.48 data[32] was used and a proper energy threshold of $100 \mathrm{eV}$ was set for tracking all particles. The major processes included in the simulation were the X-ray photoelectric process, electron ionization, and scattering. 


\subsection{Model description}

To simulate the detector physics, a detector model was established that included the geometrical construction, the corresponding physics, and incident photons properties.

In the numerical model, instrument's geometry was simplified appropriately, as demonstrated in Fig. 2(a). Electron multipliers and collimating tubes were set in the shape of thin cylinders and hollow cone shells, respectively. The critical parameters are labeled in Fig. 2(b) and an overall layout view of the instrument is demonstrated in Fig. 2(c).
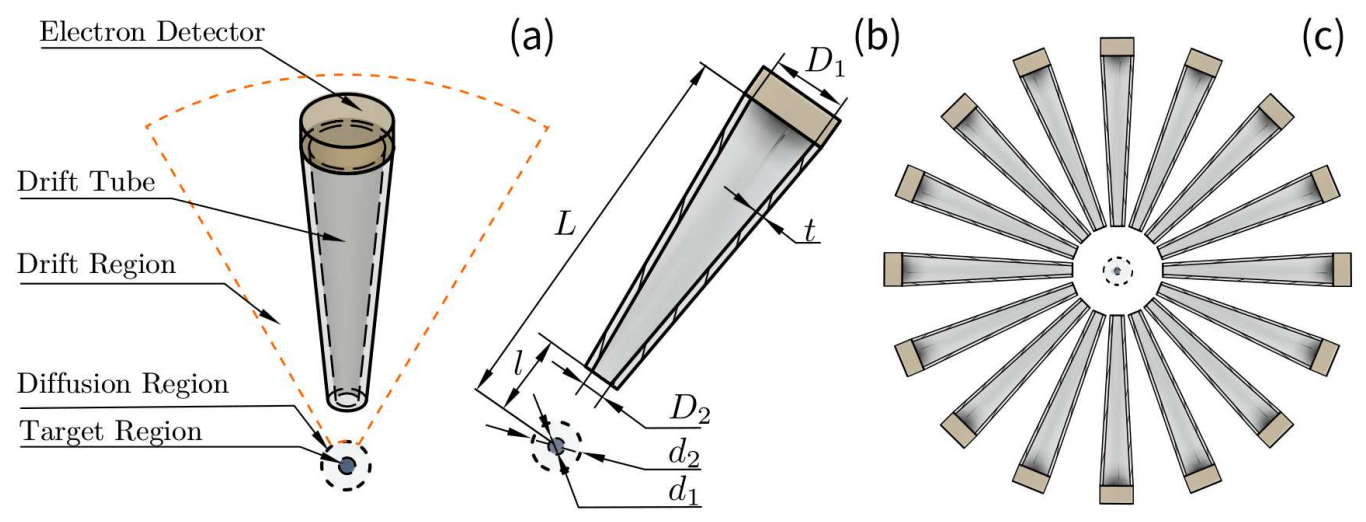

Figure 2. Sketch of e-TOF detectors and gas environment. (a) An e-TOF detector and gas regions of the spectrometer. (b) Critical parameters in design. (c) Overall layout view of e-TOF detectors.

The geometry of the differential vacuum system was omitted and the gaseous environment was divided into three parts according to the pressure magnitude: the target region, the diffusion region, and the drift region. The target region, a high-pressure cylindrical region, was located at the center of the instrument, where gas was injected and photoelectrons were produced. Since the FEL pulse duration is ultra short, the pressure in the target region was considered to be stable during a single pulse. Generally, a target gas with a higher $E_{b}$ (such as $O_{2}$ ) means a better energy resolution of $E_{p h}$ for a given drift length. However, to test the model in a moderate condition, $N_{2}\left(E_{b}=403 \mathrm{eV}\right)$ was adopted. The drift region, a steady low-pressure region for electron drifting, is used to reduce electron scattering and to satisfy the electron multipliers' working environment (in which the pressure should be $<0.1 P a$ ). To simulate the pressure transition region between the target region and the drift region, a diffusion region with 16 radius bins in linear gradient pressure was adopted in the numerical simulation. Additionally, we also considered terrestrial magnetism, which was set to be perpendicular to the detection plane, as it bends electrons maximally for such a setup.

The incident polarized X-ray photons were sampled according to the start-to-end FEL simulation under typical SXFEL working conditions, in which the FEL's average energy is $\sim 621 \mathrm{eV}$ with a narrow bandwidth of $\sim$ $0.8 \mathrm{eV}$ [13]. The photon direction was set to be perpendicular to the detection plane. In terms of photon polarity, completely polarized photons were simulated by the combination of linearly polarized light and circularly polarized light photons with a given $P_{\text {lin }}$ (determined by the FEL working conditions). The linearly polarized photon polarization angle was set to be $\Psi$, and the $\vec{E}_{c i r}$ direction for each circularly polarized photon was sampled from a uniform distribution in $[0,2 \pi]$. Meanwhile, the photon beam's transverse distribution was considered as a normal distribution. Usually, the beam diameter $\left(D_{b}\right)$ was defined as the place where photon intensity is reduced to $1 / e^{2}$ of its maximum[33], which was calculated as $4 \sigma$ of the normal distribution. 


\subsection{Simulation}

The electronics signals were generated according to the time when photoelectrons were detected and a typical pulse shape of an MCP detector. The signals were digitized by using a common sampling time of a waveform digitizer. Details are described below and simulation results are presented.

Because there are $>10^{12}$ photons in a single laser pulse, a variance reduction technique of cross-section bias for only the photoelectric process was adopted. The cross section for the photoelectric process was increased by a factor of $10^{5}$, while the other cross sections remained unchanged. Therefore, the number of total sampling events was reduced significantly, while the number of effective events remained the same.

For each X-ray FEL pulse, once photoelectrons were detected, their charge and drift time were recorded. The detectors' simulated signals were produced according to Eq. 3.2 with proper shape parameters ( $\tau$ and $\sigma_{s}$ ). The discriminator, fan in/out unit, delay cables, and a TDC unit (measuring the earliest electron's arrival time) were omitted because the e-TOF signal shape can be obtained directly in the simulation. By adopting the method mentioned above, the angular distribution can be derived by integration of the signal shape. Meanwhile, the $E_{p h}$ spectrum was derived by using pulse shape analysis.

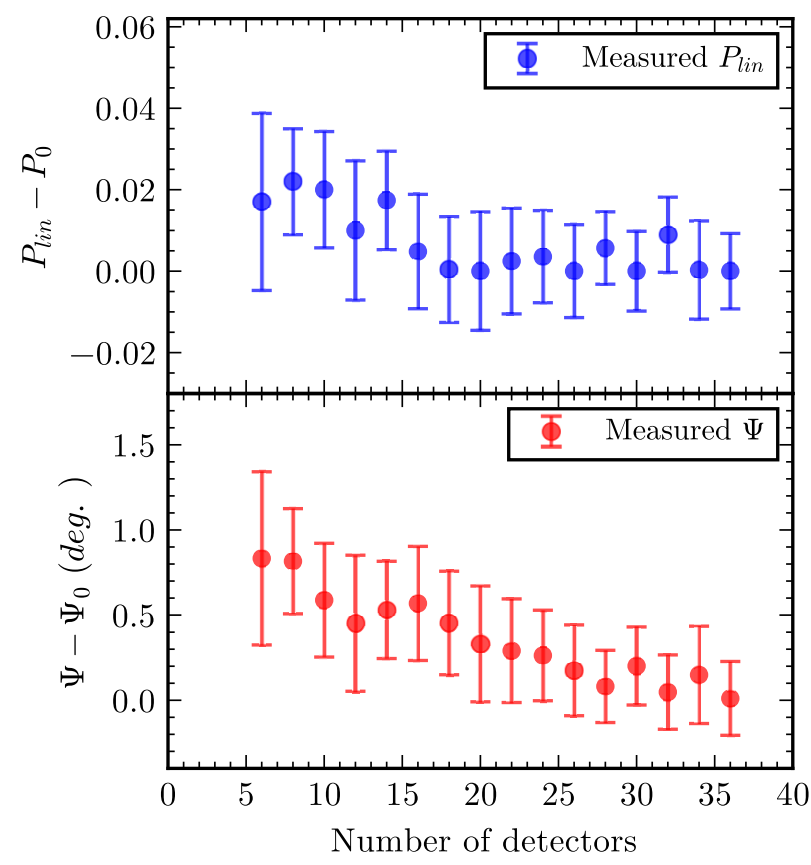

Figure 3. Performance of polarization measurement as a function of the number of detectors.

The effect of terrestrial magnetism can be corrected by various methods, such as magnetic shielding and imposition of a reversed magnetic field. Therefore, it was omitted in this simulation and the dependence on it will be studied later. Key parameters of the baseline design are listed in Table 1. It is worth mentioning that the detectors are uniformly deployed for measuring any polarization angle and that the number of detectors is determined by a balance of cost, complexity, and performance dependence analysis. Because this instrument's primary goal is to measure polarization precisely, the performance of the polarization measurement was analyzed by varying the number of detectors with the other baseline parameters being exactly same as those of Table 1. As shown in Fig. 3, one can see that the performance improvement for the polarization measurement is 
insensitive to the number of detectors when the number of detectors is $>16$. Hence, the number of detectors is fixed at 16, and the number of detectors (16) used in the previous setup [6] is also confirmed as an optimal choice by the simulations conducted in this paper.

In addition, the performance for the baseline design is shown in Fig. 4, which verifies the numerical model.

Table 1. Critical parameters of the baseline design

\begin{tabular}{|l|l|l|}
\hline Parameter & Value & Description \\
\hline$E_{p h, a v}$ & $621.4 \mathrm{eV}$ & Average energy of a typical SXFEL pulse \\
$E_{p h, \text { band }}$ & $0.9 \mathrm{eV}$ & Bandwidth of a typical SXFEL pulse \\
$N$ & 16 & Number of detectors \\
Gas & $N_{2}$ & Target gas species \\
$E_{b}$ & $403 \mathrm{eV}$ & Binding energy of $1 \mathrm{~s}$ orbit \\
$d_{1}$ & $5 \mathrm{~mm}$ & Diameter of the target region \\
$d_{2}$ & $10 \mathrm{~mm}$ & Diameter of the diffusion region \\
$D_{b}$ & $0.1 \mathrm{~mm}$ & Average diameter of the laser beam \\
$L$ & $350 \mathrm{~mm}$ & Drift distance \\
$l$ & $30 \mathrm{~mm}$ & Collimating tubes' offset \\
$D_{1}$ & $27 \mathrm{~mm}$ & Diameter of electron multipliers \\
$D_{2}$ & $3.24 \mathrm{~mm}$ & Diameter of collimation tubes' front end \\
$B$ & $0 \mathrm{gauss}$ & Terrestrial magnetic field intensity \\
$\tau$ & $200 \mathrm{ps}$ & Location parameter of detector's response for an electron \\
$\sigma_{s}$ & 0.7 & Scale parameter of detector's response for an electron \\
$t_{s}$ & $150 \mathrm{ps}$ & Sampling time interval \\
$t_{i}$ & $50 \mathrm{ps}$ & Interpolation time interval \\
\hline
\end{tabular}

\section{Dependence analysis}

Resolutions of $0.5 \%$ for the polarization degree measurement and $0.5 \mathrm{eV}$ for the $E_{p h}$ spectrum measurement are required by the SXFEL user facility, so the critical parameters' influences on the polarization and $E_{p h}$ spectrum measurements are studied in this section.

\subsection{Polarization measurement}

The precision of the polarization measurement is related to the number and angular distribution of detected photoelectrons. Potential critical factors might include the gas pressure for both the target region and the drift region, electron drift length, detector size, and the residual terrestrial magnetic field. To examine their influence, polarization measurement performance will be evaluated by using the absolute deviation and standard error of $P_{\text {lin }}$ for an incident FEL pulse with linear polarization degree $P_{0}=1$.

For the target region, a gas target with a high pressure may mean a larger photoelectron yield and a smaller statistical error term. However, at the same time, it also has a big impact photoelectron drift, resulting in a nonconvergent measurement. Accordingly, the pressure needs to be optimized, and the related simulation results are shown in Fig. 5, indicating an optimal $P_{t}$ in the range from 0.1 to $1.0 \mathrm{~Pa}$. 
(a)

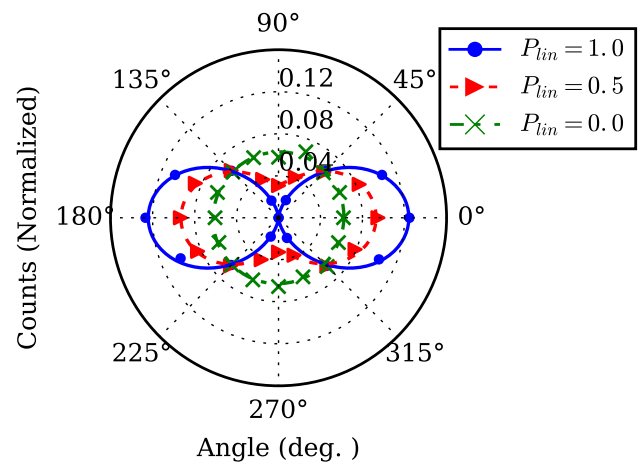

(b)

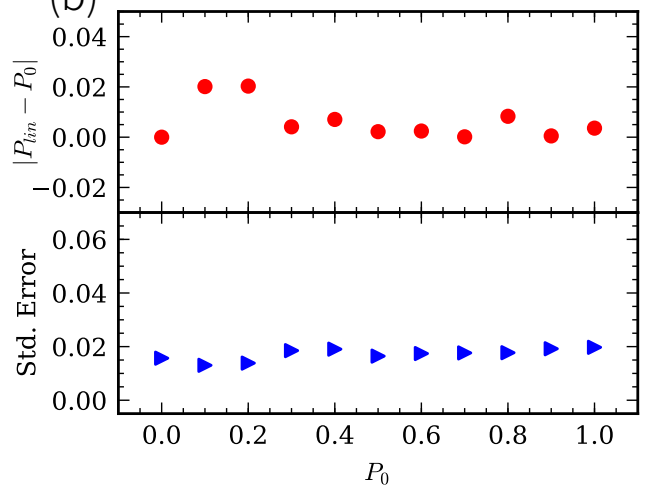

(c)

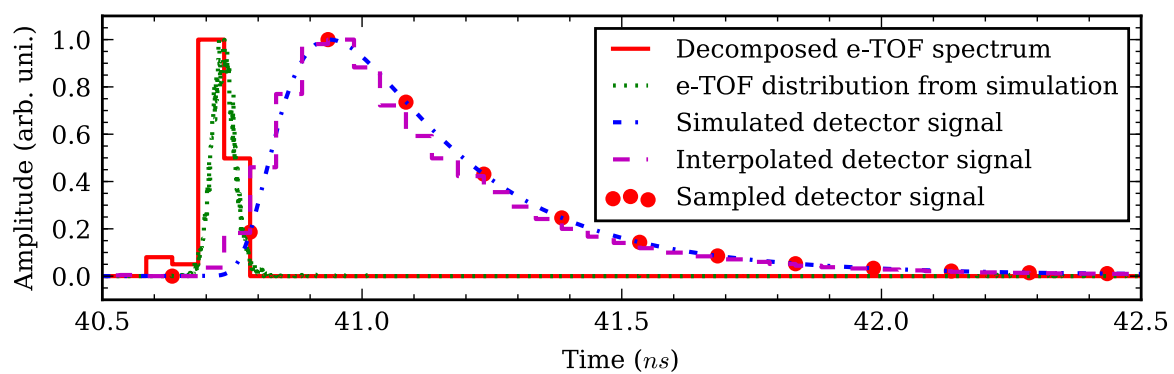

(d)

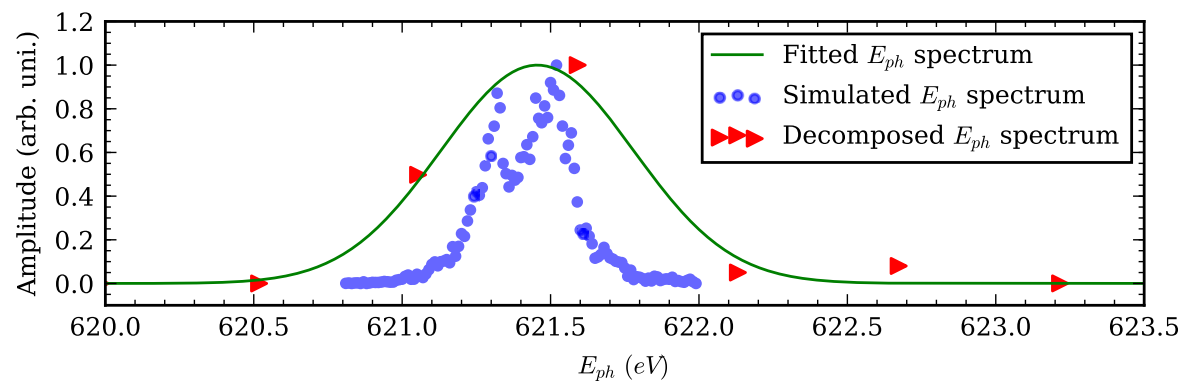

Figure 4. Simulation results for the baseline design. (a) Simulated photoelectron angular distribution for an X-ray FEL with a linear polarization angle of zero degrees in the polar coordinate system as a function of $P_{\text {lin }}$. (b) Absolute deviation of polarization measurement for different linear polarization fractions $P_{0}$ in the simulation. (c) Decomposed e-TOF spectrum from detector signals, which is compared with a realistic electron TOF distribution. The simulated e-TOF signal (dashed and dotted curve) was sampled by the waveform digitizer in $\Delta t_{s}=150 \mathrm{ps}$ and interpolated with $\Delta t_{i}=50 \mathrm{ps}$. After offline analysis, the decomposed e-TOF spectrum (solid curve) is capable of describing the e-TOF distribution from the simulation (dotted curve). (d) Comparison between the decomposed energy spectrum (fitted by a normal distribution) and the $E_{p h}$ spectrum from the start-to-end simulation of an X-ray FEL.

For the drift region, a gas environment with high pressure prevents photoelectron drift. As shown in Fig. 6, when $P_{d}<0.1 P a$, deviations of the measured $P_{\text {lin }}$ change insignificantly and satisfy the corresponding design requirements.

A longer drift length indicates a larger statistical error owing to the smaller acceptance angle. As can be inferred from Fig. 7, as drift distance increases, $\left|\Delta P_{\text {lin }}\right|$ changes slightly. Therefore, an acceptable drift length might be from 200 to $400 \mathrm{~mm}$ to satisfy the design requirement.

Electron detectors with smaller size might lead to greater statistical error owing to their small acceptance angle, while larger detectors might cause error term because of the unsuitable assumption of the detection plane 


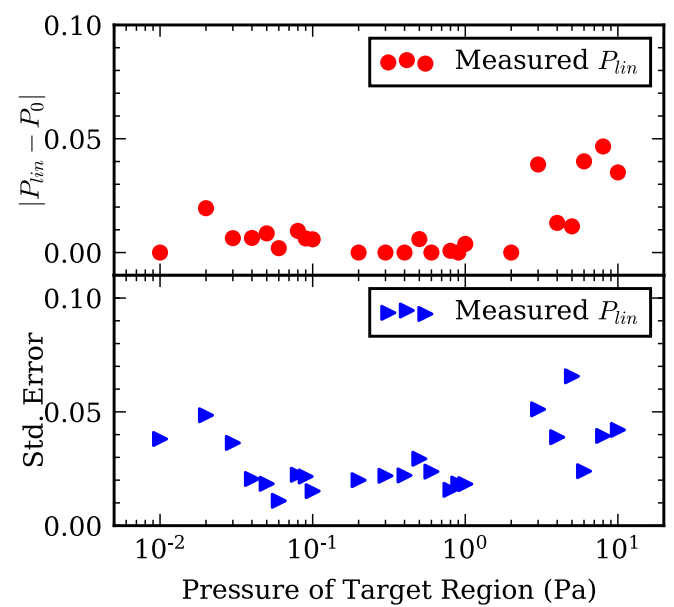

Figure 5. Deviation of polarization measurement with changing pressure in the target region.

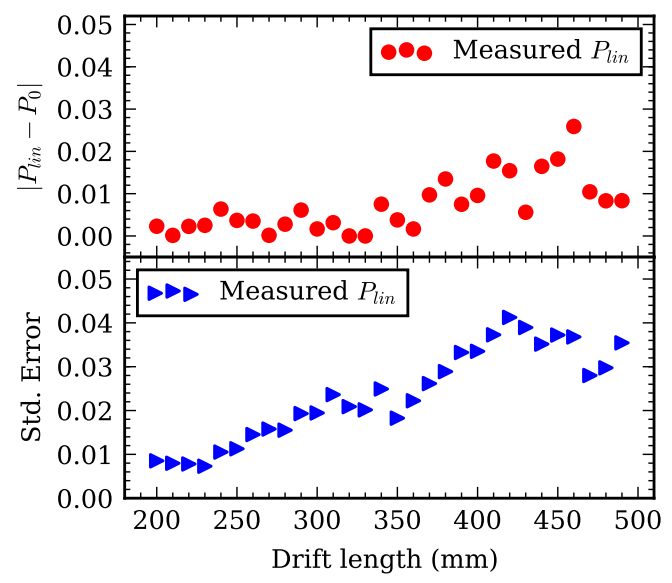

Figure 7. Deviation of polarization measurement with changing drift distance.

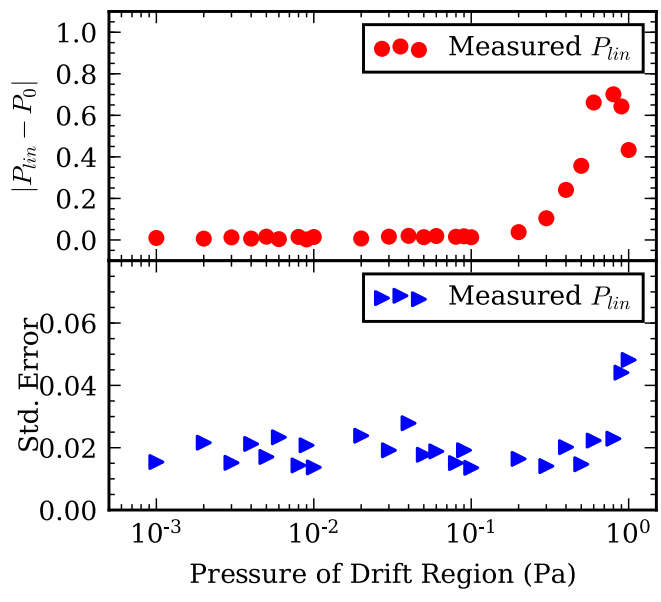

Figure 6. Deviation of polarization measurement with changing pressure in the drift region.

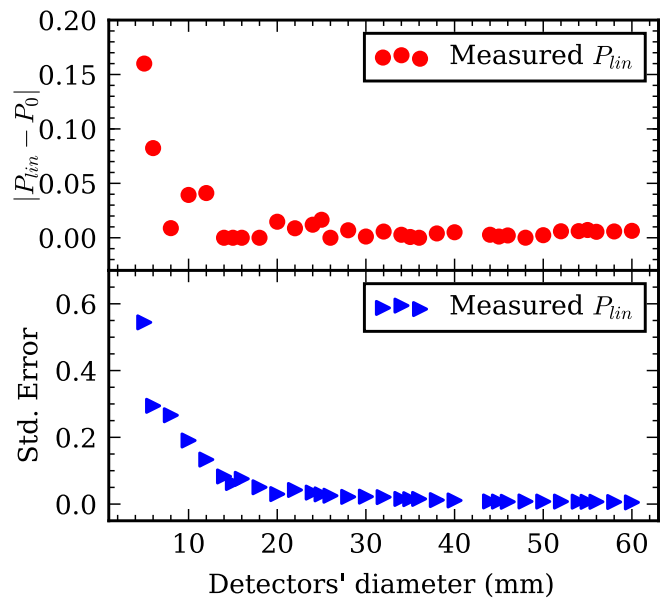

Figure 8. Deviation of polarization measurement with changing detector size.

and inaccurate positioning. The result is demonstrated in Fig. 8. From the result, detected photoelectrons can be regarded as a point source and to be within the detection plane when the detectors' diameter $\left(D_{2}\right)$ is in the range from 5 to $60 \mathrm{~mm}$ and the drift length $L$ is $350 \mathrm{~mm}$. Meanwhile, $D_{2}$ should be $>30 \mathrm{~mm}$ (typically $42 \mathrm{~mm}$ for commercial products) to comply with the design requirement.

The terrestrial magnetic field bends the drift path of the photoelectrons, resulting in deviations for measurement. Such deviations can be avoided by magnetic shielding or imposing a reversed magnetic field; however, how weak the residual magnetic field should be is a question to be answered by us. The magnetic field in any direction can be decomposed into orthogonal directions : perpendicular $\left(B_{\perp}\right)$ and parallel $\left(B_{/ /}\right)$to the detection 

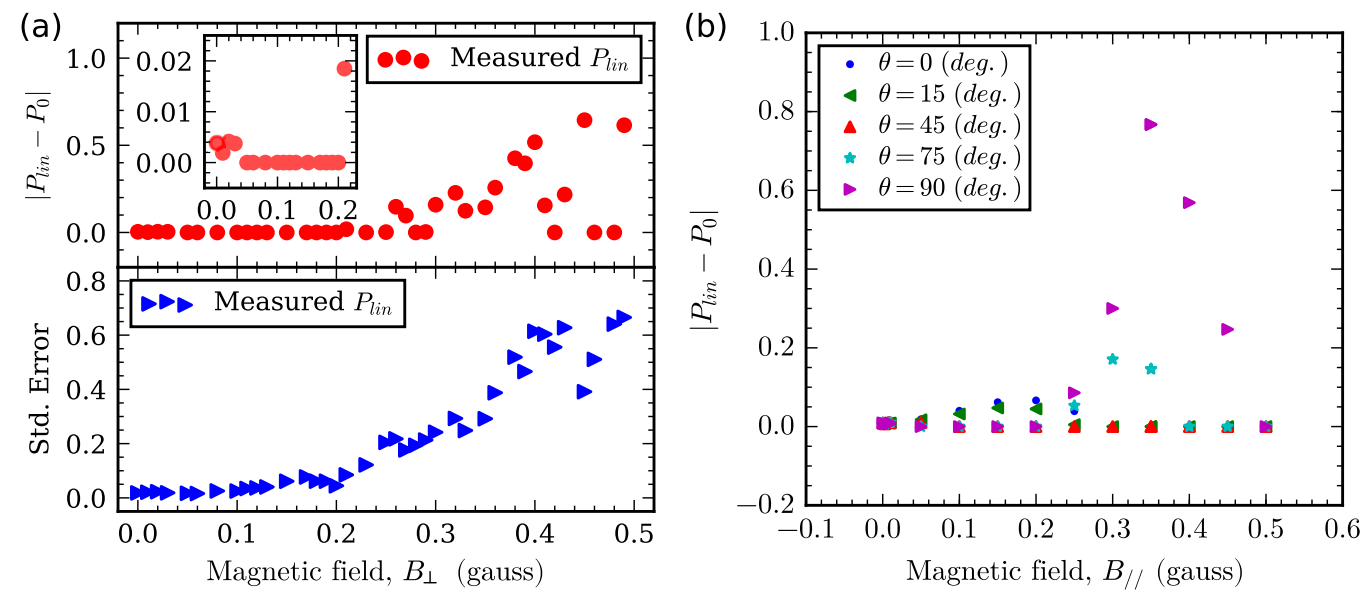

Figure 9. Deviation of polarization measurement with changing residual Earth magnetic field in the direction (a) perpendicular to the detection plane and (b) parallel to the detection plane, respectively.

plane. The influence of the perpendicular component is same for photoelectrons at any direction in detection plane, while the influence of the parallel component is different for photo-electrons in different directions. So the dependence of the polarization measurements on the residual terrestrial magnetic field for these two directions was studied. As shown in Fig. 9, a maximum residual magnetic field of $\sim 0.2$ gauss in perpendicular direction for any linear polarization angle and in parallel direction for polarization angle of about $45^{\circ}$ is small enough to ignore its influence. For $B_{/ /}$with other linear polarization angles, a more strict magnetic shielding is demanded to improve measurement precision. The terrestrial magnetic declination and the magnetic inclination at SXFEL site are $-5^{\circ} 52^{\prime}$ and $46^{\circ} 52^{\prime}$, respectively [34]. Additionally, the designed beam direction and linear polarization of SXFEL are about $11^{\circ}$ south of west and almost horizontal in detection plane, respectively. Based on these facts, further simulations were performed to evaluate the influence of residual magnetic field for real situation, showing a maximum residual magnetic field of $\sim 0.2$ gauss can meet the requirement of measurement precision and higher magnetic shielding is strongly required for other linear polarization angles.

\section{$5.2 E_{p h}$ spectrum measurement}

For the $E_{p h}$ spectrum measurement, longer signal duration and smaller sampling and interpolation time intervals are preferred, both of which mean higher energy resolution. A longer signal duration can be achieved by a longer drift length $(L)$ and by using a target gas with a higher binding energy. The sampling time interval $\Delta t_{s}$ is often determined by the maximum sampling rate of the waveform digitizer. Larger $\Delta t_{s}$ means worse precision of sampled signals, which leads to distortion in later interpolation. Moreover, the interpolation time interval $\Delta t_{i}$ should be chosen according to the full width at half maximum, because two overlapped signal peaks can be mistaken as one peak. In the following, we study the following key parameters: species of target gas, drift length $L$, and sampling and interpolation time intervals ( $\Delta t_{s}$ and $\Delta t_{i}$, respectively). To examine the performance of measuring the $E_{p h}$ spectrum, the primary X-ray energy in a normal distribution with $\mu=621 \mathrm{eV}$ (average) and $\sigma=0.1 \mathrm{eV}$ (deviation) was simulated. The estimated parameters ( $\hat{\mu}$ and $\hat{\sigma}$ ) can be obtained by fitting the decomposed signal with a normal distribution. Since $\hat{\mu}$ can be corrected by using another advanced spectrometer, $\hat{\sigma}-\sigma$ might be the figure of merit (FOM) for evaluating spectrometer performance.

According to Eq. 2.5, a target gas with a higher binding energy means a lower photoelectron kinetic energy, which results in longer signal duration for a given drift length and furthermore a higher resolution when other 
parameters remain the same. Both nitrogen and oxygen are acceptable and their binding energies are $\sim 403$ and $\sim 540.43 \mathrm{eV}$, respectively [26]. The result of the $E_{p h}$ spectrum measurement simulations for these two kinds of target gases are demonstrated in Fig. 10. According to the simulation, $\hat{\sigma}$ for the $\mathrm{O}_{2}$ target is much smaller than that for $\mathrm{N}_{2}$. Therefore, $\mathrm{O}_{2}$ is more suitable for the $E_{p h}$ spectrum measurement.
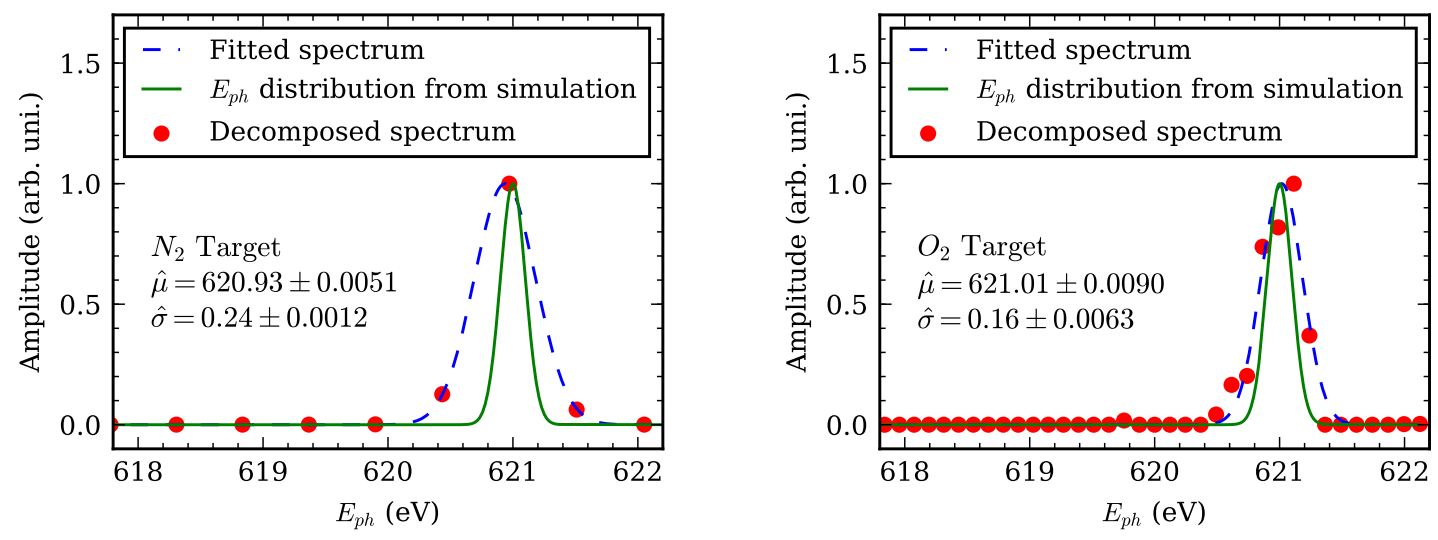

Figure 10. Simulation result for the $N_{2}$ target (left) and the $O_{2}$ target (right) with a sampling time interval of $\Delta t_{s}=100$ (ps) and a resampling time interval of $\Delta t_{i}=50(p s)$.

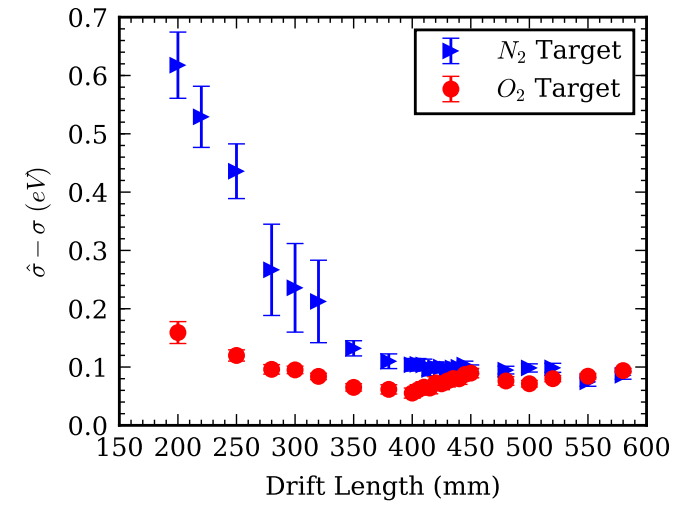

Figure 11. Deviation of $\hat{\sigma}$ with changing drift distance. 


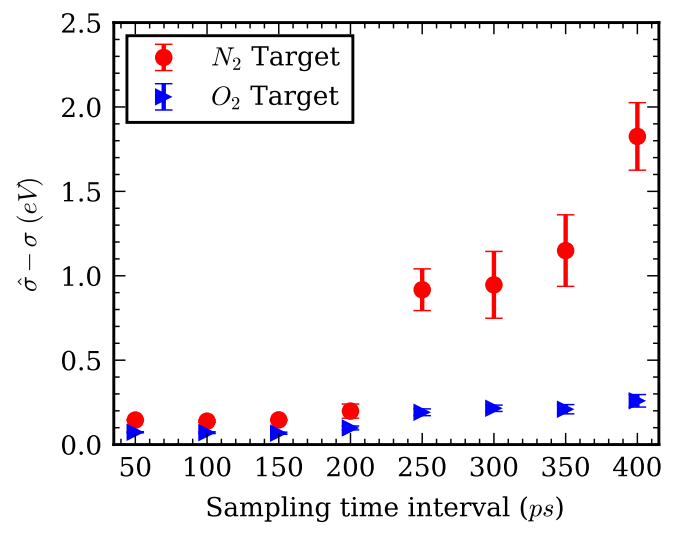

Figure 12. Deviation of $\hat{\sigma}$ with changing sampling time interval $\Delta t_{s}$.

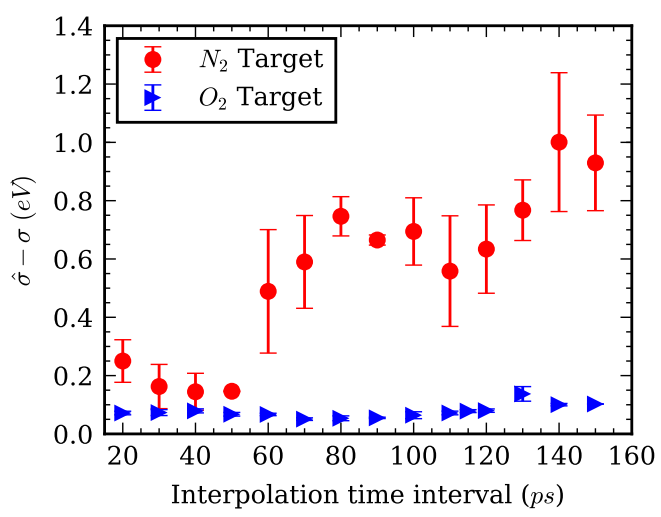

Figure 13. Deviation of $\hat{\sigma}$ with changing interpolation time interval $\Delta t_{i}$.

A longer drift length converts small energy differences of electrons into measurable time and helps to extend the signal duration for digitizer sampling. The simulation result for the dependence of drift distance $L$ is reported in Fig. 11. From the result, it can be inferred that $L$ should be $\geq 350 \mathrm{~mm}$ for $\mathrm{N}_{2}$. In contrast, for the $\mathrm{O}_{2}$ target, the smallest deviation of $\hat{\sigma}$ is located at $L \sim 400 \mathrm{~mm}$, which can be understood because a longer drift length means fewer detected photoelectrons while a shorter drift length results in a shorter flight time. In short, the $O_{2}$ target is more suitable than $N_{2}$ and $L=400 \mathrm{~mm}$ for the $O_{2}$ target is the optimal design.

Nowadays, analog-to-digital converter sampling rates are as high as $30 \mathrm{Gs}$ [35]. Generally, $t_{s}$ from 50 to $400 \mathrm{ps}$ is practicable and acceptable. By changing $\Delta t_{s}$ and $\Delta t_{i}$, the variation of $\hat{\sigma}$ can be demonstrated, as shown in Figs. 12 and 13. According to Figs. 12 and 13, $\hat{\sigma}$ for the $O_{2}$ target is better than that for $N_{2}$ when both $\Delta t_{s}$ and $\Delta t_{i}$ are the same. Moreover, for the $O_{2}$ target, the design requirement can be satisfied as long as $\Delta t_{s}$ and $\Delta t_{i}$ are within the simulation range (i.e., $\Delta t_{i}<\Delta t_{s}$ is required).

In addition, the same method for energy spectrum reconstruction was used to separate photons with two energies from the same X-ray FEL pulse under optimized conditions $\left(E_{p h 1}=621 \mathrm{eV}, E_{p h 2}=621 \mathrm{eV}+\Delta E\right.$, and $\Delta t_{s}=150 \mathrm{ps}$ for an $O_{2}$ target). The scan was performed by changing $\Delta E$ and the two energy peaks were obvious when $\Delta E \geq 0.3 \mathrm{eV}$, giving an energy resolution of $0.3 \mathrm{eV}$

\section{Discussion and conclusion}

Based on the previous design concept of an e-TOF instrument [6] and the numerical optimization by using simulation tools, we have validated the feasibility of an e-TOF-based polarimeter and spectrometer for an X-ray FEL user facility. The polarization resolution is $0.5 \%$ and the photon's energy resolution is $0.3 \mathrm{eV}$ for the optimized design and the following requirements are obtained:

1. The target region's pressure must be in the range from 0.1 to $1.0 \mathrm{~Pa}$ and the drift region's pressure must be $<0.1 P a$.

2. The drift distance $L$ must be $>400 \mathrm{~mm}$.

3. The diameter of the detectors must be $>30 \mathrm{~mm}$. 
4. The remanent magnetic field must be controlled to be $<0.2$ gauss.

5. The sampling time interval must be in the range from 50 to $400 \mathrm{ps}$ and the interpolation time interval must be in the range from 20 to $140 \mathrm{ps}$.

There are still a few conditions to be considered for further study, such as the influence on $P_{\text {lin }}$ from photoelectrons from $p$ subshells and the smaller effect of Auger electrons on the polarity measurement.

\section{Acknowledgments}

This work is supported by the Fundamental Research Funds for the Central Universities (Grant No. xjj2017109), the National Natural Science Foundation of China (Grant No. 11775293), the Natural Science Fundamental Research Plan of Shaanxi Province (Grant No. 2016JM1019), the China Association for Science and Technology, and the Ten Thousand Talents Program.

\section{References}

[1] B. W. J. McNeil and N. R. Thompson, X-ray free-electron lasers, Nature Photonics 4 (2010) 814-821.

[2] A. Singer, I. A. Vartanyants, M. Kuhlmann, S. Duesterer, R. Treusch and J. Feldhaus, Transverse-coherence properties of the free-electron-laser FLASH at DESY, Physical Review Letters 101 (2008) 2-5.

[3] F.-e. L. Sacla, X.-r. Regime and F. Lehmku, Single Shot Coherence Properties of the Free-Electron Laser SACLA in the Hard X-ray Regime, .

[4] C. Behrens, F.-J. Decker, Y. Ding, V. A. Dolgashev, J. Frisch, Z. Huang et al., Few-femtosecond time-resolved measurements of X-ray free-electron lasers, Nature Communications 5 (apr, 2014) 1-7.

[5] H. Deng, T. Zhang, L. Feng, C. Feng, B. Liu, X. Wang et al., Polarization switching demonstration using crossed-planar undulators in a seeded free-electron laser, Physical Review Special Topics - Accelerators and Beams 17 (feb, 2014) 020704.

[6] E. Allaria, B. Diviacco, C. Callegari, P. Finetti, B. Mahieu, J. Viefhaus et al., Control of the polarization of a vacuum-ultraviolet, high-gain, free-electron laser, Physical Review X 4 (2014) .

[7] A. A. Lutman, J. P. MacArthur, M. Ilchen, A. O. Lindahl, J. Buck, R. N. Coffee et al., Polarization control in an $X$-ray free-electron laser, Nature Photonics 10 (jul, 2016) 468-472.

[8] Emma P., Akre R., Arthur J., Bionta R., Bostedt C., Bozek J. et al., First lasing and operation of an angstrom-wavelength free-electron laser, Nature Photonics 4 (sep, 2010) 641-647.

[9] T. Ishikawa, H. Aoyagi, T. Asaka, Y. Asano, N. Azumi, T. Bizen et al., A compact X-ray free-electron laser emitting in the sub-angstrom region, Nature Photonics 6 (aug, 2012) 540-544.

[10] A.S.Schwarz and European XFEL Group, the European X-Ray Free Electron Laser Project At Desy, Proc. 2004 FEL Conf. (2004) 85.

[11] R. P. Walker, D. Bulfone, F. Cargnello, M. Castellano, F. Cevenini, F. Ciocci et al., The FERMI FEL project at Trieste, Nuclear Instruments and Methods in Physics Research, Section A: Accelerators, Spectrometers, Detectors and Associated Equipment 375 (1

[12] Z. T. Zhao, S. Y. Chen, L. H. Yu, C. X. Tang, L. X. Yin, D. Wang et al., SHANGHAI SOFT X-RAY FREE ELECTRON LASER TEST FACILITY, in IPAC, (San Sebastián, Spain), pp. 3011-3013, 2011.

[13] M.-H. Song, C. Feng, D.-Z. Huang, H.-X. Deng, B. Liu and D. Wang, Wakefields studies for the sxfel user facility, Nuclear Science and Techniques 28 (May, 2017) 90. 
[14] L. Feng, H. Deng, T. Zhang, C. Feng, J. Chen, X. Wang et al., Single-shot measurement of free-electron laser polarization at SDUV-FEL, Chinese Physics C 39 (2015) 028101.

[15] P. Finetti, M. Zangrando, G. D. Ninno, J. Buck, C. Svetina, S. D. Mitri et al., Polarization measurement of free electron laser pulses in the vuv generated by the variable polarization source fermi, Proceedings of SPIE - The International Society for Optical Engineering 9210 (2014) 273-274.

[16] A. J. Nelson, J. Dunn, T. Van Buuren and J. Hunter, X-ray laser-induced photoelectron spectroscopy for single-state measurements, Applied Physics Letters 85 (2004) 6290-6292.

[17] M. Saes, F. van Mourik, W. Gawelda, M. Kaiser, M. Chergui, C. Bressler et al., A setup for ultrafast time-resolved $x$-ray absorption spectroscopy, Review of Scientific Instruments 75 (2004) .

[18] A. M. March, A. Stickrath, G. Doumy, E. P. Kanter, B. Krässig, S. H. Southworth et al., Development of high-repetition-rate laser pump/x-ray probe methodologies for synchrotron facilities,

Review of Scientific Instruments 82 (2011).

[19] R. W. Schoenlein, S. Chattopadhyay, H. H. W. Chong, T. E. Glover, P. A. Heimann, C. V. Shank et al., Generation of Femtosecond Pulses of Synchrotron Radiation, Science 287 (2000) 2237-2240.

[20] X. Zhang, G. Smolentsev, J. Guo, K. Attenkofer, C. Kurtz, G. Jennings et al., Visualizing Interfacial Charge Transfer in Ru-Dye-Sensitized TiO 2 Nanoparticles Using X-ray Transient Absorption Spectroscopy, The Journal of Physical Chemistry Letters 2 (mar, 2011) 628-632.

[21] C. Bressler, C. Milne, V.-T. Pham, A. ElNahhas, R. M. van der Veen, W. Gawelda et al., Femtosecond XANES Study of the Light-Induced Spin Crossover Dynamics in an Iron(II) Complex, Science 323 (2009) 489-492.

[22] S. Agostinelli, J. Allison, K. Amako, J. Apostolakis, H. Araujo, P. Arce et al., Geant4âĂ ̌̆a simulation toolkit, Nuclear Instruments and Methods in Physics Research Section A: Accelerators, Spectrometers, Detectors and Associated Equipment 506 (20

[23] J. W. Cooper, Photoelectron-angular-distribution parameters for rare-gas subshells, Physical Review A 47 (1993) 1841-1851.

[24] S. T. Manson and A. F. Starace, Photoelectron angular distributions: energy dependence for s subshells for $s$ subshells, Reviews of Modern Physics 54 (1982) 389-405.

[25] A. Paulus, C. Winterfeldt, T. Pfeifer, D. Walter, G. Gerber and C. Spielmann, Novel time-of-flight electron spectrometer optimized for time-resolved soft-x-ray photoelectron spectroscopy, Review of Scientific Instruments 77 (2006) 0-6.

[26] M. B. Trzhaskovskaya, V. G. Yarzhemsky and V. I. Nefedov, Photoelectron angular distribution parameters for elements $z=1$ to $z=54$ in the photoelectron energy range $100 \hat{A} \breve{S} 5000 \mathrm{ev}$, Atomic Data and Nuclear Data Tables 77 (jan, 2001) 97-159.

[27] M. Trzhaskovskaya, V. Nefedov and V. Yarzhemsky, Photoelectron angular distribution parameters for elements $z=$ 55 to $z=100$ in the photoelectron energy range 100-5000 ev, Atomic Data and Nuclear Data Tables 82 (2002) 257-311.

[28] M. Yamazaki, J.-I. Adachi, T. Teramoto and A. Yagishita, Experimental evidence of interatomic resonant Auger electron emission from fixed-in-space NO molecules, Journal of Physics B: Atomic, Molecular and Optical Physics 40 (2007) 207-213.

[29] S. Jetter, D. Dwyer, W.-Q. Jiang, D.-W. Liu, Y.-F. Wang, Z.-M. Wang et al., PMT waveform modeling at the Daya Bay experiment, Chinese Physics C 36 (2012) 733-741.

[30] M. R. Hestenes and E. Stiefel, Methods of conjugate gradients for solving linear systems, Journal of Research of the Natural Bureau of Standards 49 (1952) 409-436, [1102 .0183].

[31] D. C. Liu and J. Nocedal, On the limited memory BFGS method for large scale optimization, Mathematical Programming 45 (aug, 1989) 503-528. 
[32] C. Champion, S. Incerti, H. Aouchiche and D. Oubaziz, A free-parameter theoretical model for describing the electron elastic scattering in water in the Geant4 toolkit, Radiation Physics and Chemistry 78 (2009) 745-750.

[33] A. E. Siegman, How to (maybe) measure laser beam quality, in DPSS (Diode Pumped Solid State) Lasers: Applications and Issues, p. 6, Optical Society of America, 1998.

[34] "Magnetic-Declination.com." http://www.magnetic-declination.com. Accessed: 2017-08-21.

[35] X. Zhu, D. Wu, L. Zhou, Y. Huang, J. Wu and X. Liu, A four-channel time-interleaved 30-gs/s 6-bit adc in 0.18 $\hat{\text { Iijm }}$ sige bicmos technology, Science China Information Sciences 60 (2017) 129401. 